\title{
SENSITIVITY AND SPECIFICITY OF MRI IN DETECTING MALIGNANT SPINAL CORD COMPRESSION AND IN DISTINGUISHING MALIGNANT FROM BENIGN COMPRESSION FRACTURES OF VERTEBRAE
}

\author{
King C. Li* and Peter Y. Poon $†$ \\ Departments of Radiology, †Ontario Cancer Institute and University of Toronto, \\ and *University of Michigan Medical Centre
}

\begin{abstract}
The accuracy of magnetic resonance (MR) imaging in the detection of metastatic compression of the spinal cord and the cauda equina (MCCE) in 75 patients with known primary malignancy outside the central nervous system is determined retrospectively by comparing the MR results with findings of myelography, surgery, clinical follow-up and autopsy. The sensitivity is $93 \%$, the specificity $97 \%$ and the overall accuracy $95 \%$. The signal intensity measured in the sagittal MR images of a collapsed vertebral body is divided by that of an average of three adjacent normal vertebrae to form a signal intensity ratio (SIR). The SIRs of 41 metastatic and 15 post-traumatic collapsed vertebrae are calculated. A ratio of 0.8 has the most differentiating power. All benign and one malignant compressed vertebrae have SIRs greater than 0.8 .
\end{abstract}

Keywords: Cancer (metastatic to spine); Collapsed vertebrae; MRI; Spinal cord compression; Trauma.

\section{INTRODUCTION}

Patients with metastatic compression of the spinal cord and the cauda equina (MCCE) may experience a variety of symptoms and signs such as pain along the spine, radicular pain, motor weakness, sensory deficit, extensor plantar response, bowel or urinary incontinence and vertebral tenderness. ${ }^{5}$ However, these symptoms and signs may also be due to a number of non-compressive etiologies including paraneoplastic syndromes such as carcinomatous myopathy or neuropathy, radiation myelopathy, herpes zoster, subacute necrotic myelopathy, pain from a pelvis or long bone metastasis, anterior spinal artery occlusion, retroperitoneal tumor or toxicity of cytotoxic agents. In addition, non-malignant diseases such as herniated intervertebral disc, osteoporotic vertebral fracture and intraspinal abscess may also cause cord compression in a cancer patient. Therefore, the development of a sensitive and specific test for distinguishing compressive causes will be most useful in the management of cancer patients. With the advent of magnetic resonance (MR) imaging, we have a highly sensitive means of working up patients with myelopathy and detecting vertebral body metastases. ${ }^{1,2,3,6,15}$ However, the precise value of MR in detecting MCCE and in distinguishing malignant from benign compression fracture has not been reported. Wc therefore undertook a retrospective study to determine the sensitivity and specificity of MR in (a) detecting MCCE using complete myelography, clinical follow-up, surgical findings and autopsy as the gold standard, and (b) distinguishing malignant from benign compression fracture of vertebrae.

\section{MATERIALS AND METHODS}

From January 1986 to March 1987,86 patients with known malignancies outside the central nervous system had magnetic resonance (MR) imaging of the spine at the Ontario Cancer Institute for the investigation of possible metastatic compression of the spinal cord and the cauda equina (MCCE). The MR examinations were done using a resistive 0.15 Tesla imager. Contiguous $1.0 \mathrm{~cm}$ thick sagittal and transverse images were taken using spin echo pulse sequences with a repetition time (TR) of $1030 \mathrm{~ms}$ and an
RECEIvEd 1/21/88; ACCEPTED 2/26/88.

Acknowledgment-The authors thank Mrs. Patricia Field and Mrs. Irene Silva for typing the manuscript and Miss Doris Moro, Mrs. Katherine MacKay and Miss Catherine Macewko for performing the MR examinations.
Please address all correspondence to Dr. Peter Y. Poon, Department of Diagnostic Imaging, St. Michael's Hospital, 30 Bond Street, Toronto, Ontario, M5B IW8 Canada. 
echo time (TE) of $30 \mathrm{~ms}$. Two series of data acquisition in each plane were needed to cover the full length of the entire spine with a body coil which has a core of $54 \mathrm{~cm}$. When the most median sagittal section was not obtained, the examination was repeated with an off-set of $0.5 \mathrm{~cm}$. Sometimes a $20 \mathrm{~cm}$ surface coil was also used to study the focal region of interest and the slice thickness could be reduced to $0.5 \mathrm{~cm}$, achieving better spatial resolution. The criteria used in this study to define MCCE were (a) partial or complete loss of the low signal intensity space (combination of CSF, meninges and cortical bone of the vertebrae) surrounding the spinal cord and the cauda equina, (b) displacement, flattening or complete loss of definition of the spinal cord (Fig. 1). Both authors interpreted the MR images without knowledge of the clinical status of the patients. A disagrecment was resolved by reviewing the images and discussion.

The medical and imaging records of these 86 patients were reviewed to identify two groups of patients:

\section{(1) MCCE Group}

Patients with documented reliable evidence (gold standard) of presence or absence of MCCE are in-

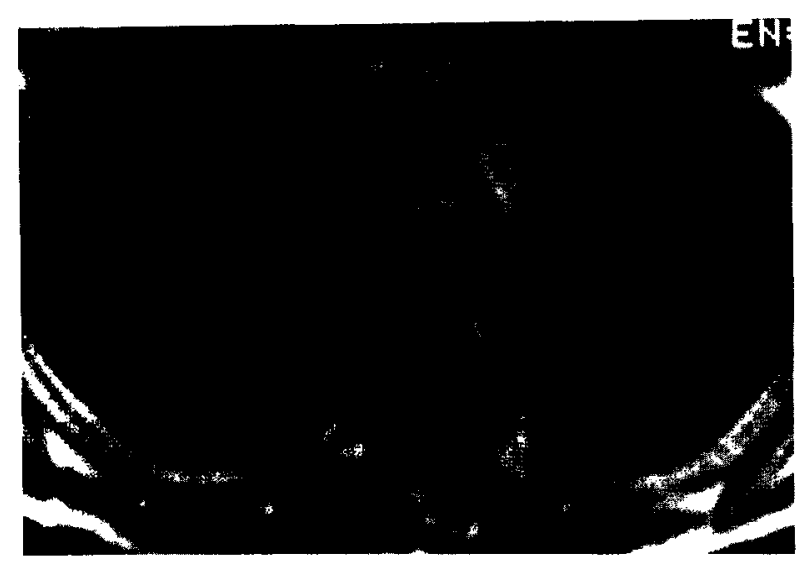

A

Fig. 1. MR criteria for cord compression. (A) and (B) are transverse and sagittal MR images obtained by SE 1000/30. The vertebral body of D5 (arrow) has a lower signal intensity than that of others. It is collapsed and bulges posteriorly, flattening the spinal cord (curved arrow) obliquely. The right anterior low signal intensity space around the spinal cord is lost. cluded in this group. The gold standard including myelographic, surgical, autopsy and compelling clinical findings was used to assess the accuracy of the MR imaging results, and

\section{(2) Collapsed Vertebra Group}

This group includes patients with collapsed vertebral bodies which were shown to be due to metastatic disease by surgery, needle biopsy, plain radiography and/or radionuclide scintigraphy.

MCCE group. Twenty-six patients had a myelogram within two weeks of the MR scans. The complete myelogram was performed by a lumbar puncture with $12 \mathrm{cc}$ of Pantopaque (Alcon Laboratories Inc., Humacao, Puerto Rico) injected into the subarachnoid space. Pantopaque was used instead of a water soluble contrast agent because, when cord compression was detected, repeated examinations could be done without multiple lumbar punctures after treatment. The entire subarachnoid space was examined from the sacral region up to the foramen magnum. When a complete blockage was found and surgical decompression was intended, a Cl-C2 puncture would also be done to determine the upper limit of the obstruction. The myelographic films and the original

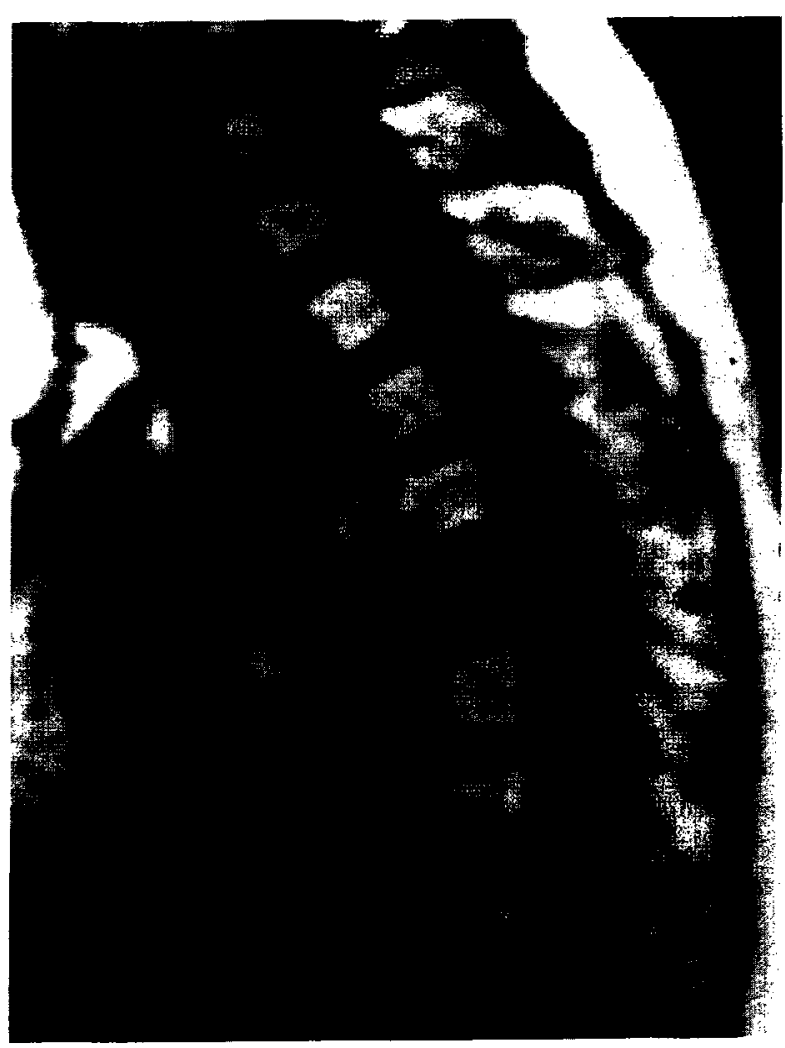

B 
reports were reviewed. Any distortion and/or displacement of the contrast column as well as incomplete or complete blockage of the flow of contrast were recorded.

Ten patients had a complete myelogram and a surgical decompression. Laminectomy of the affected vertebrae was done. The compressing tissue was removed, providing pathological evidence of MCCE. Another five patients had surgical decompression only, without having been investigated by myelography. Two patients died within two months after the MR examinations. The spinal cord and the spine were searched for metastatic disease at autopsy. Myelography had not been performed in these two patients.

Thirty-two patients had adequate clinical follow-up information in their medical records to indicate with reasonable certainty that MCCE was or was not present at the time of MR examination. If within a month of the MR examination a patient's original neurological symptoms and signs indicating cord compression did not deteriorate or require any additional investigations, he or she was considered not to have MCCE. On the other hand, MCCE was judged to be present if the same symptoms and signs improved after having been treated by local irradiation or deteriorated where irradiation had not been given.

Out of the 86 patients, a group of 75 was identified. The MR examinations of this group of patients were of satisfactory quality and independent evidence was available to determine whether MCCE was present or not. The results of MR in these patients were compared with the independent clinical or pathological evidence. There were 49 females and 26 males and their ages ranged from 39 to 77 years old with a mean of 51. The primary neoplasms are listed in Table 1 .

Collapsed vertebrae group. Forty-six malignant collapsed vertebral bodies were identified in $32 \mathrm{pa}$ -

Table 1. Primary Neoplasms

Breast carcinoma
Prostate carcinoma
Lymphoma
Multiple myeloma
Renal cell carcinoma
Bronchogenic carcinoma
Malignant melanoma
Endometrial carcinoma
Thyroid carcinoma
Parathyroid carcinoma
Laryngeal carcinoma
Colonic carcinoma
Chondrosarcoma
Cardinoma of vulva

tients among the entire group of 86 . The metastatic nature was established by surgical pathology in $9 \mathrm{pa}-$ tients and needle biopsy results in 2 patients. In the remaining 21 patients, the plain radiographs of the spine and radionuclide scintigraphs showed obvious metastatic collapsed vertebrae. In addition, these patients also had cerebral, pulmonary or hepatic metastases. In three patients with five collapsed vertebral bodies, the metastatic involvement was diffuse and normal vertebral bodies could not be identified. Using an electronic cursor of the biggest possible size, the signal intensity in the sagittal images obtained by the body coil of these 41 compressed vertebral bodies was measured. The signal intensity of three relatively normal vertebrae adjacent to each of the compressed ones was also recorded. The signal intensity ratio (SIR) of a metastatic and compressed vertebrae was then calculated. To minimize partial volume effect, only the median sagittal images were used.

For comparison, the MR scans of 11 patients with 15 post-traumatic compressed vertebral bodies, referred to the University of Michigan Medical Center for evaluation of post-traumatic myelography, were reviewed. Contiguous sagittal sections, $5 \mathrm{~mm}$ thick, were obtained in 9 patients and $10 \mathrm{~mm}$ thick contiguous sagittal sections were obtained in 2 patients using a superconducting imager operating at $0.35 \mathrm{~T}$ and a body coil. Pulse sequences used included $\mathrm{TR}=0.5 \mathrm{sec}, \mathrm{TE}=28-56 \mathrm{~ms}, n=2 ; \mathrm{TR}=0.5 \mathrm{sec}$, $\mathrm{TE}=40 \mathrm{~ms}, n=8 ; \mathrm{TR}=1.5 \mathrm{sec}, \mathrm{TE}=28-56 \mathrm{~ms}$, $n=1 ; \mathrm{TR}=1.5 \mathrm{sec}, \mathrm{TE}=40-80 \mathrm{~ms}, n=1$. The SIRs of compressed versus normal vertebral bodies at the first echo of the different pulse sequences were calculated. The second echo images were not used for the quantitative analysis because of the poor sensitivity of $T_{2}$-weighted images in detecting bone marrow abnormalities. ${ }^{6}$

Since, depending on the slice thickness, there can be two to five sagittal images of a vertebra, more than one SIR may be calculated for the same vertebra. In the group of 41 malignant collapsed vertebrae, 51 SIRs wcre obtained. Among the 15 post-traumatic compressed vertebrae, 85 ratios were calculated. The large number of SIRs in the latter group was due to the fact that multiple pulse sequences had been used and most of the scans had a thickness of $5 \mathrm{~mm}$.

\section{RESULTS}

The results are first analysed according to the gold standard groupings. Table 2 shows the comparison between MR and the gold standard. In the first group of 26 patients where myelogram served as the yardstick, 
Table 2. Correlation of MRI with Gold Standard Groups

\begin{tabular}{lcc}
\hline & \multicolumn{2}{c}{ MRI } \\
\cline { 2 - 3 } Gold Standard & Definite MCCE & No MCCE \\
\hline Complete myelography & & 1 \\
$n=26$ & & 4 \\
Definite MCCE & & \\
$\quad$ No MCCE & & \\
Complete myelography and & & \\
surgical decompression & & \\
$n=10$ & 10 & \\
Definite MCCE & & \\
No MCCE & & \\
Surgical decompression & & \\
$n=5$ & 5 & 2 \\
Definite MCCE & & \\
No MCCE & & \\
Autopsy & & \\
$n=2$ & & \\
Definite MCCE & & \\
No MCCE & & \\
Clinical follow-up & & \\
$n=32$ & & \\
Definite MCCE & & \\
No MCCE & & \\
\hline
\end{tabular}

Table 3. Comparison of MRI with Gold Standard

\begin{tabular}{lcc}
\hline & \multicolumn{2}{c}{$\begin{array}{c}\text { Myelography/Surgery/ } \\
\text { Autopsy/Clinical Findings }\end{array}$} \\
\cline { 2 - 3 } MRI & Definite MCCE & No MCCE \\
\hline Definite MCCE & 41 & 1 \\
No MCCE & 3 & 30 \\
\hline
\end{tabular}

MR and myelography agreed with each other in 24 patients (Fig. 2) with only one falsc-negative and one false-positive MR interpretation. Six patients in this group also had two sites of MCCE and C1-C2 puncture was needed to demonstrate the second lesion in three patients. MR showed all these locations of compression very well in one examination. A total of 15 patients had surgical decompression. MR demonstrated accurately the full extent of MCCE and, where myelography had been performed (four had both lumbar and C1-C2 punctures), MR provided comparable information (Fig. 3). There is $100 \%$ agreement between MR interpretation and the surgical and autopsy findings (Fig. 4) with no false-positive or false-negative results in these 17 patients. In the last group where clinical follow-up provided the "truth" data, there were two false-negative MR interpretations.

The overall results of the entire group of 75 patients are shown in Table 3 . The sensitivity is $93 \%$ $(41 / 44)$, the specificity $97 \%(30 / 31)$ and the accuracy $95 \%(71 / 75)$. The positive predictive value is $98 \%$ $(41 / 42)$ and the negative predictive value is $91 \%$ (30/33). There are one false-positive (Fig. 5) and three false-negative MR interpretations. The clinical and imaging details of these four patients are summarized in Table 4.

The signal SIRs of 15 benign and 41 malignant collapsed vertebrae are shown in Figure 6. Only one metastatic collapsed vertebra has a ratio greater than 0.8 whereas all post-traumatic ones have ratios greater than 0.80 . Therefore, using 0.8 as the discriminating ratio, $97.6 \%$ of the malignant collapsed vertebrae could be distinguished from the benign ones, and none of the benign collapsed vertebrae would be misdiagnosed as malignant. The sensitivity is $97.6 \%$

Table 4. Summary of False-positive and False-negative MR Interpretations

\begin{tabular}{|c|c|c|c|}
\hline Patient/Diagnosis & MR Findings & Gold Standard & Status \\
\hline A.S. Breast carcinoma & $\begin{array}{l}\text { Loss of anterior half of low } \\
\text { signal intensity space and cord } \\
\text { is slightly flattened at D4 }\end{array}$ & Normal myelography & False-positive \\
\hline C.T. Prostate carcinoma & $\begin{array}{l}\text { Decreased anterior low signal } \\
\text { intensity space but cord not } \\
\text { displaced at D12 }\end{array}$ & Incomplete myelographic block at D12 & False-negative \\
\hline A.F. Breast Carcinoma & No MCCE & $\begin{array}{l}\text { Clinical follow-up showed increasing } \\
\text { weakness of both lower extremities and } \\
\text { the weakness improved after irradiation } \\
\text { to T10 to L3 }\end{array}$ & False-negative \\
\hline S.J. Prostate carcinoma & $\begin{array}{l}\text { Examination limited to lower } \\
\mathrm{T} \text { and L spine, showed com- } \\
\text { pression of cauda equina. } \\
\text { Patient declined further } \\
\text { examination of cervical spine }\end{array}$ & $\begin{array}{l}\text { Before MR examination, patient had } \\
\text { lumbar pain. Five days later there was } \\
\text { loss of power of all four extremities } \\
\text { indicating compression of cervical cord }\end{array}$ & False-negative \\
\hline
\end{tabular}




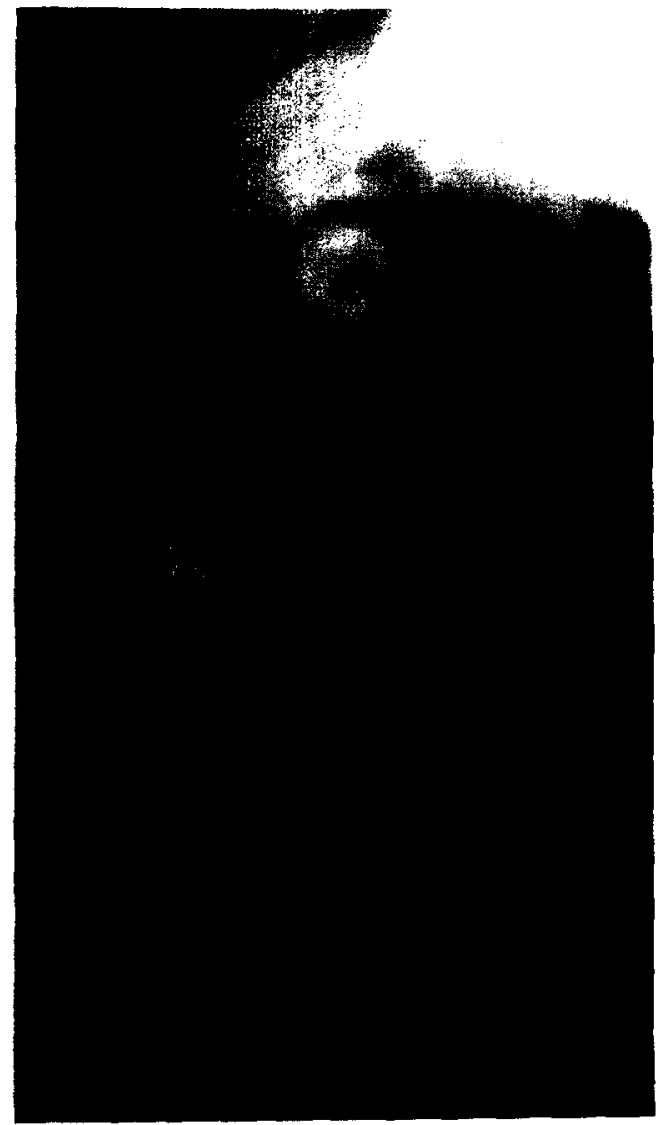

A

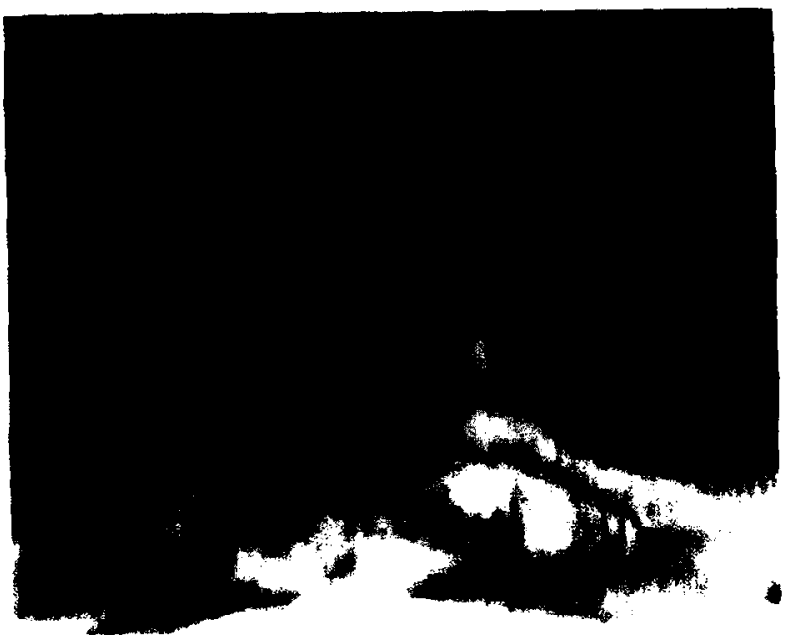

C

Fig. 2. (A) and (B) are lateral radiographs taken during myelography. The contrast medium flows towards the head in (A) and towards the feet in (B). The displaced contrast column (arrow) outlines the extradural compression at D4. (C) and (D) are transverse and sagittal MR images obtained by SE 1030/30 with a surface coil. In (C), the low signal intensity space is markedly reduced, particularly posteriorly. The cord (arrow) is displaced towards the left by the expanded right pedicle. In (D), the metastatic collapsed D4 (arrow) compresses the cord.

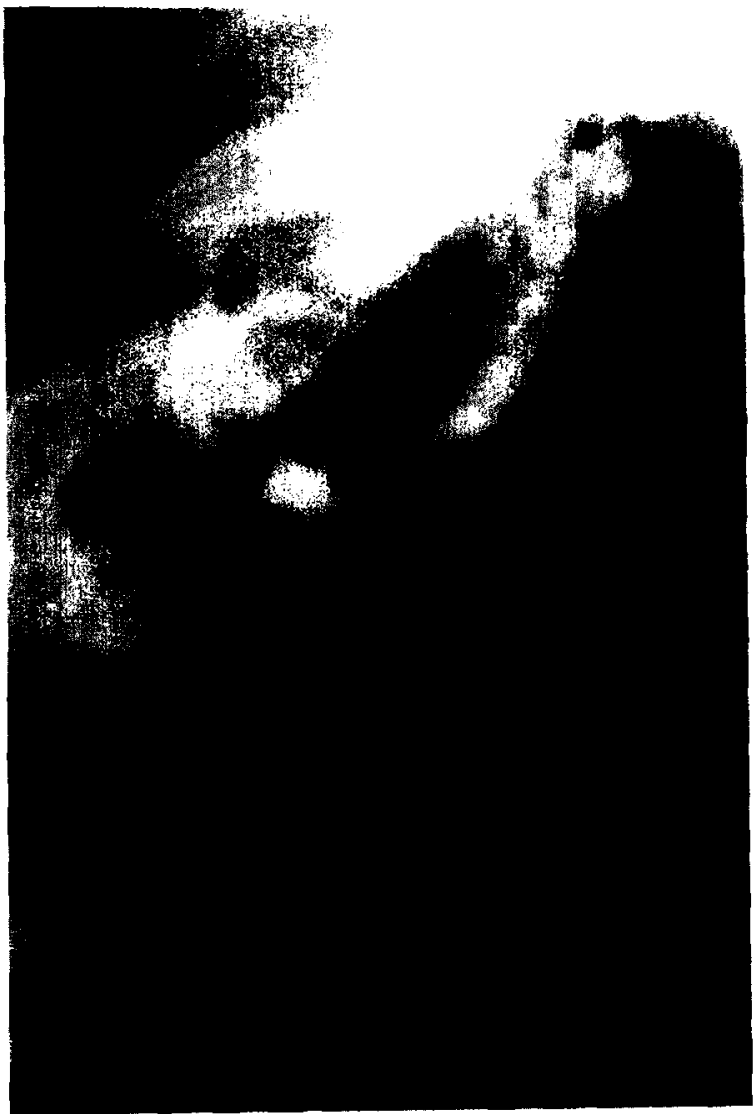

$\mathbf{B}$

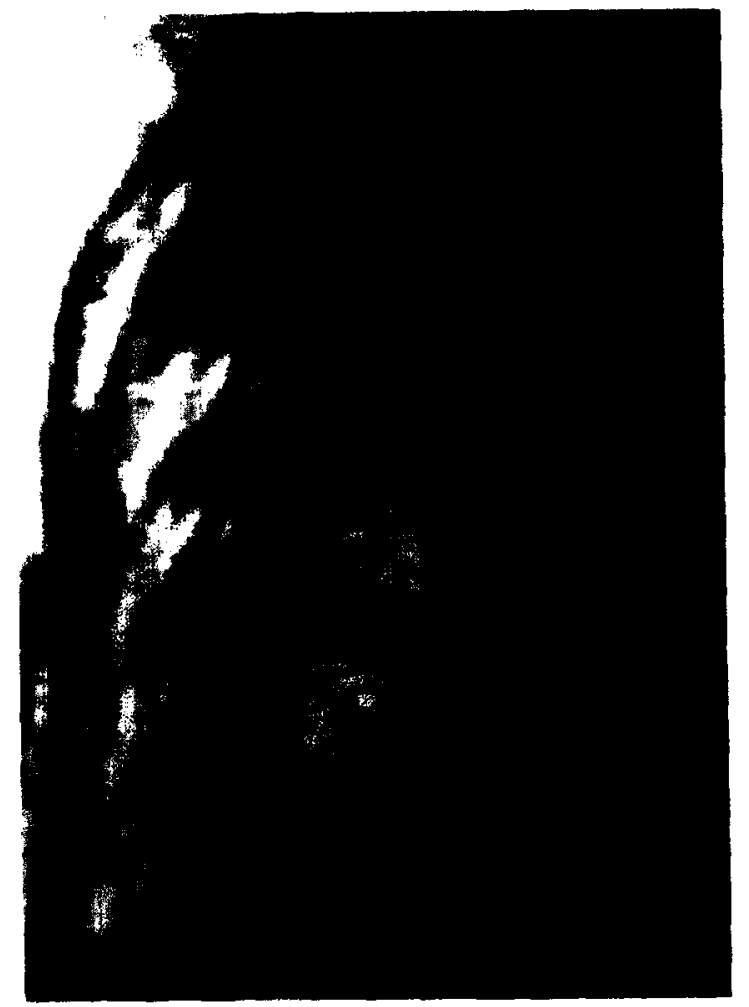

D 


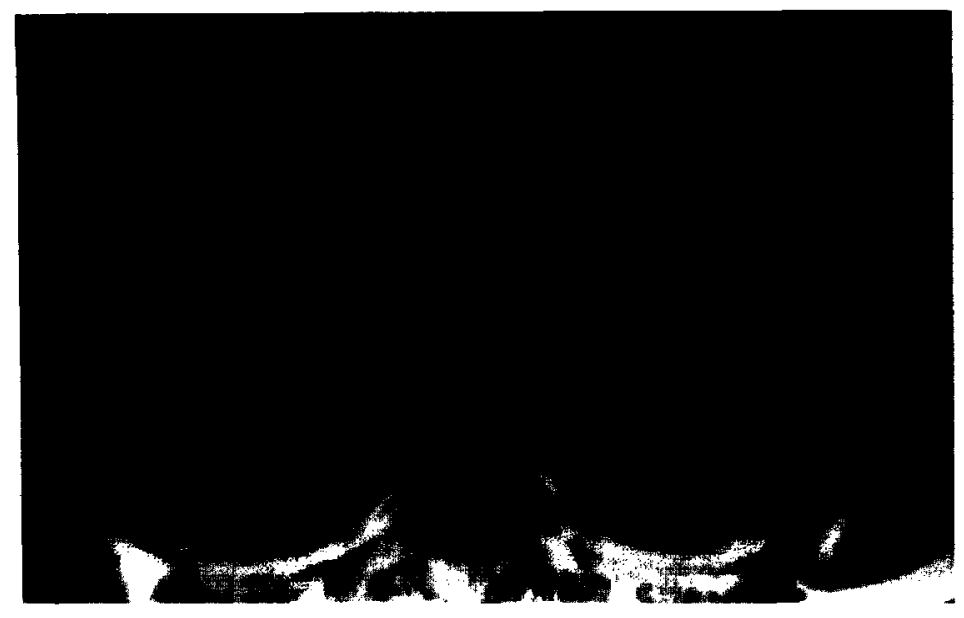

A

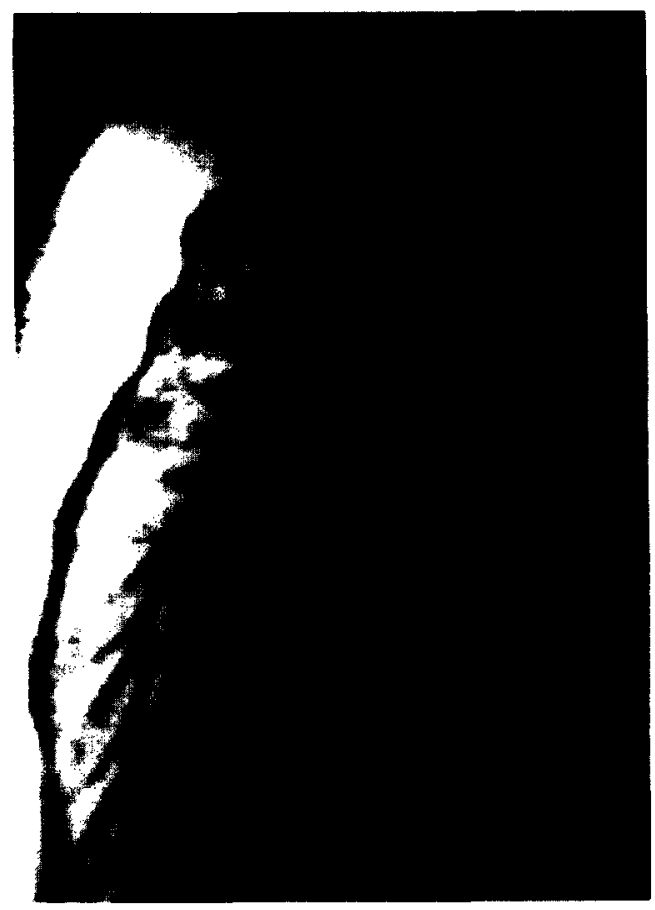

B
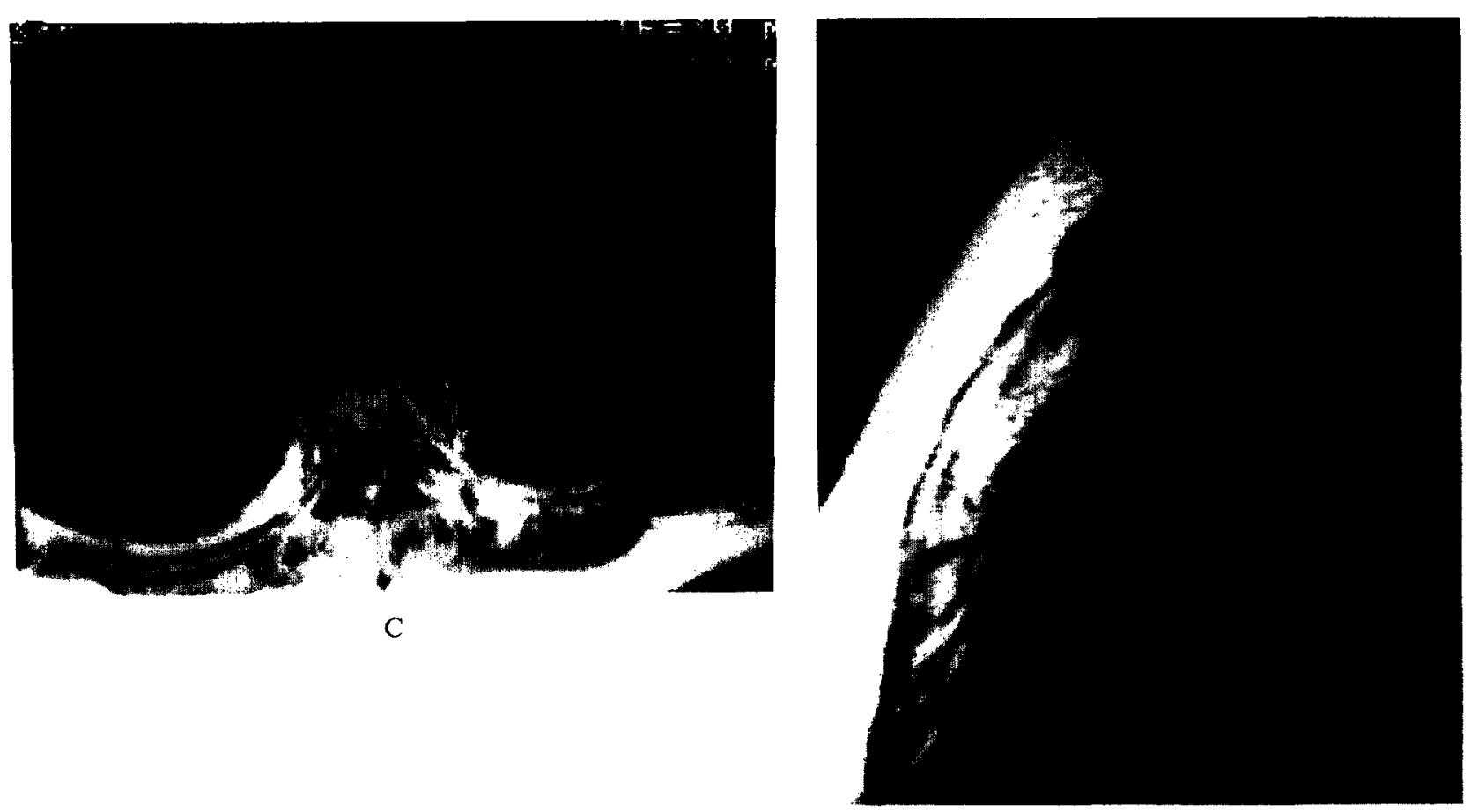

$\mathrm{D}$

Fig. 3. (A) and (B) are transverse and sagittal sections obtained by SE 1030/30 with a surface coil. The cord compression at D5 in this patient with a metastatic malignant melanoma is characterized by loss of the anterior low signal intensity space and flattening of the cord. The myelogram done later in the same day showed an incomplete block at this level. The patient was treated by irradiation. However, four weeks later the pain was worsc than bcforc. Rc-screening myelogram showed a complete blockage at the same level. A repeat MR (C) and (D) with the same technique revealed more collapse of D5 (arrow) and medium signal intensity (curved arrow) in the spinal canal obscuring the cord. Paraspinal and medastinal disease is also observed. Surgical decompression was done and metastatic tumor was present in the bone and the spinal canal. 


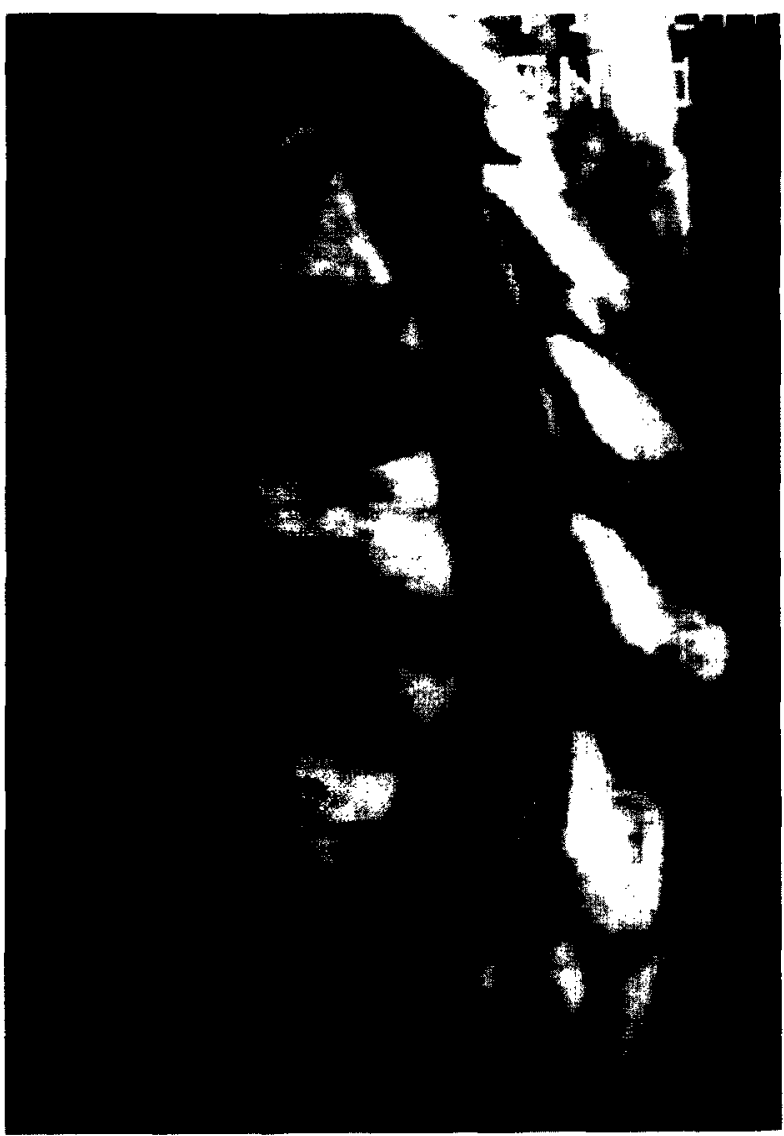

Fig. 4. The sagittal image is obtained by SE $1030 / 30$ with a surface coil. Many of the dorsal vertebrae are severely compressed especially D11 (arrow). However, the signal intensity of D11 is not reduced. A sharp, pointed edge is pressing the cord. This is a non-malignant mechanical compression. The patient died one month afterwards and there was no metastatic tumor in the bone or in the spinal canal at autopsy.
$(40 / 41)$, the specificity is $100 \%(15 / 15)$ and the accuracy $98.2 \%(55 / 56)$.

Of interest are two patients with breast carcinoma who had multiple collapsed vertebral bodies (Fig. 7). These compressed vertebrae could be divided into two groups according to whether their signal intensity ratios were bigger or smaller than 0.8 . This quantitative division correlated very well with clinical findings, plain radiographs and radionuclide scintigraphy such that the ratio of 0.8 did discriminate malignant and benign causes of the collapsed vertebral bodies.

\section{DISCUSSION}

MR had been shown to be very useful in many spinal disorders. ${ }^{7-11}$ Our data demonstrated that MR is also efficacious in the work-up of patients suspected to have MCCE because of its high sensitivity $(93 \%)$ and specificity $(97 \%)$ values. MR has a distinct advantage over myelography because no uncomfortable positionings or contrast injections are required. $\mathrm{Pa}$ tients with a complete or partial block of the spinal canal from an epidural metastasis will often have additional epidural deposits at other levels. ${ }^{12,14}$ MR did show multiple sites of epidural metastases in six patients in this study. In four of them the additional sites of involvement were not evident in the initial myelogram via a lumbar puncture and required a separate myelographic study via a $\mathrm{C} 1-\mathrm{C} 2$ puncture for demonstration. Such information is extremely valuable in choosing a plausible and most beneficial treatment. Multiple sites of involvement almost always preclude surgical intervention but radiation can be guided for complete coverage of all locations of disease to provide optimal palliative treatment.

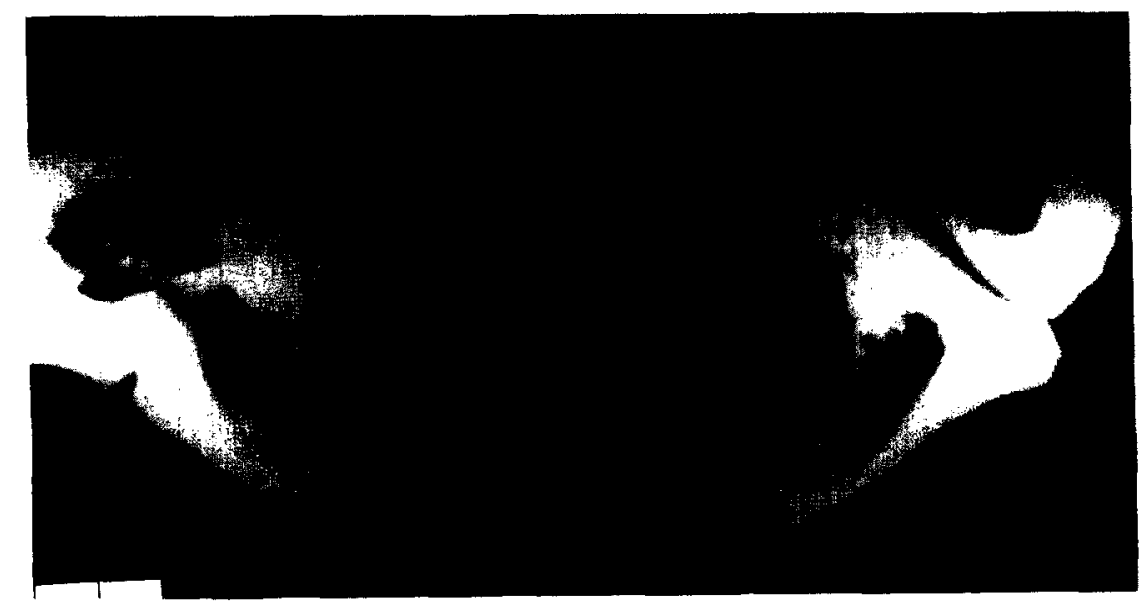

Fig. 5. A transverse MR section (SE 1030/30) at D4 shows loss of anterior low signal intensity space and slightly flattening of the cord. Myelogram performed 2 days before was normal. 


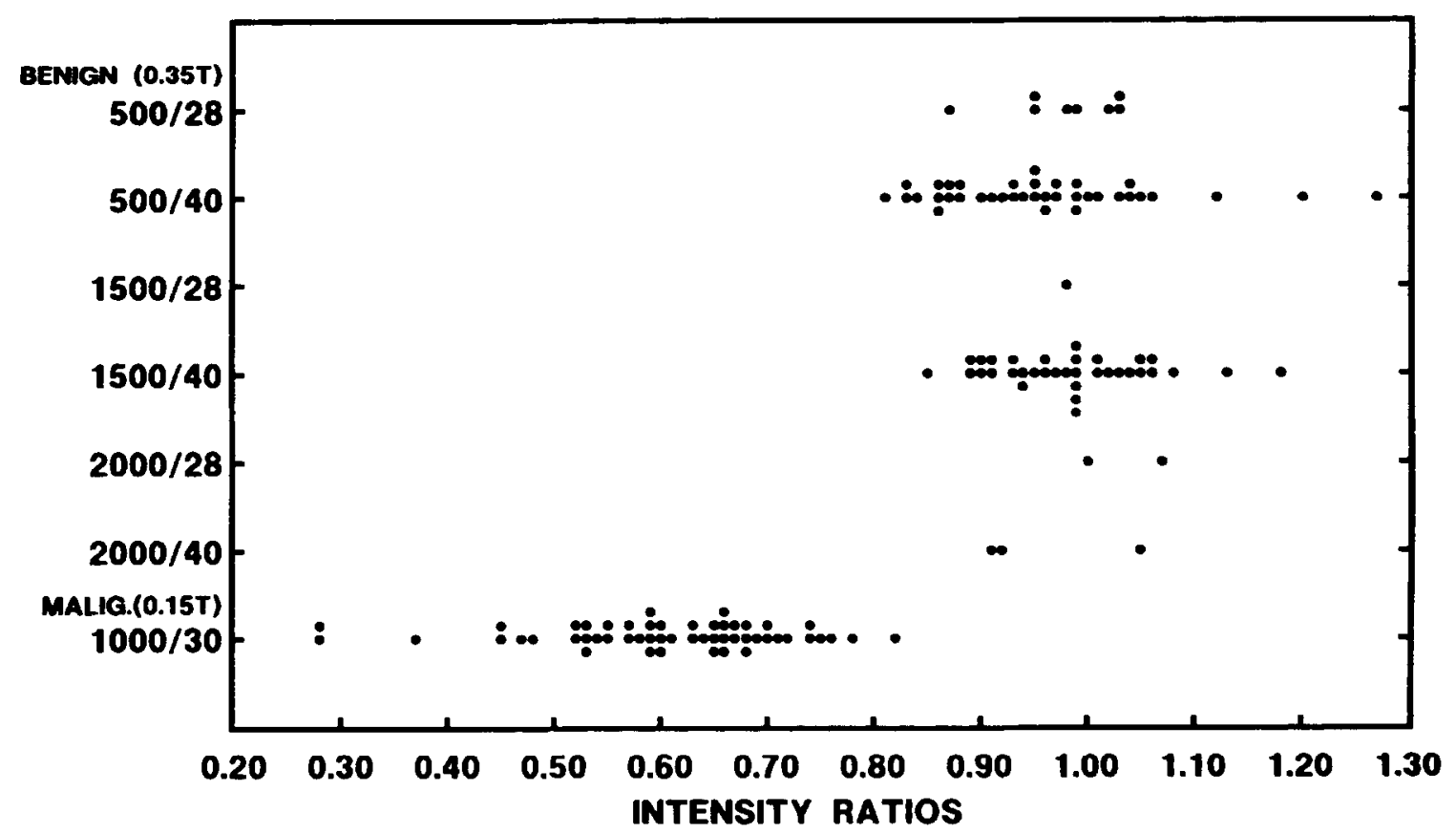

Fig. 6. Distribution of signal intensity ratios of benign and malignant collapsed vertebrae according to pulse sequences.

It is not surprising that the correlation between the MR findings and the surgical results in 15 patients was $100 \%$. This group of patients is highly selective. The patients were clearly symptomatic with definite neurological signs and symptoms. The metastatic disease in the spine and MCCE were obvious in the images.

The MR criteria used in this study to define cord compression are basically morphological signs. With the limited spatial resolution of our resistive 0.15 Tesla imager, it is understandable that subtle or marginal spinal cord displacement may be misinterpreted or missed. The detection of cord displacement becomes less certain and more subjective when the spine is scoliotic. This can account for the false-positive and two of the three false-negative MR results listed in Table 4. Images obtained at higher magnetic field, that is, 1.5 Tesla, have much improved morphologic details and will eliminate such inaccuracy. We had used the surface coil more frequently in the recent months to improve the image details when the spinal disease could be localized. The third false-negative result serves to emphasize the importance of a complete examination, covering the entire spine and its contents.

Using a $T_{2}$-weighted pulse sequence, high signal intensity in the compressed cord parenchyma has been attributed to cord edema. ${ }^{3}$ This "signal aberration," if indeed is due to localized edema as a result of compression, may increase the accuracy of MR interpretation in patients where there are minimal cord displacement and only partial obliteration of the low signal intensity space around the cord. We were not able to evaluate this potential criterion in our study because of the technical limitations of our imager at a low magnetic field.

Collapsed vertebral bodies are not infrequent findings in patients with cancer. Metastatic disease is a prime consideration but other causes, particularly osteoporotic vertebral fractures, have to be excluded. The differentiation becomes critical when management decision depends on whether there is metastatic disease or not. It is apparent from our data that MR is very accurate $(98 \%)$ in distinguishing malignant from benign collapsed vertebral bodies. However, the actual accuracy may be lower than indicated in our study because most of the cancer patients studied had advanced widespread bony metastatic disease. The collapsed vertebrae were either moderately or severely involved by metastases. Such status is desirable to ensure that the imaging diagnosis is correct since pathological confirmation is not available in all patients. When less severely involved vertebral bodies are compared, there may be more overlap between the benign and malignant groups. ${ }^{13}$ Further prospective 


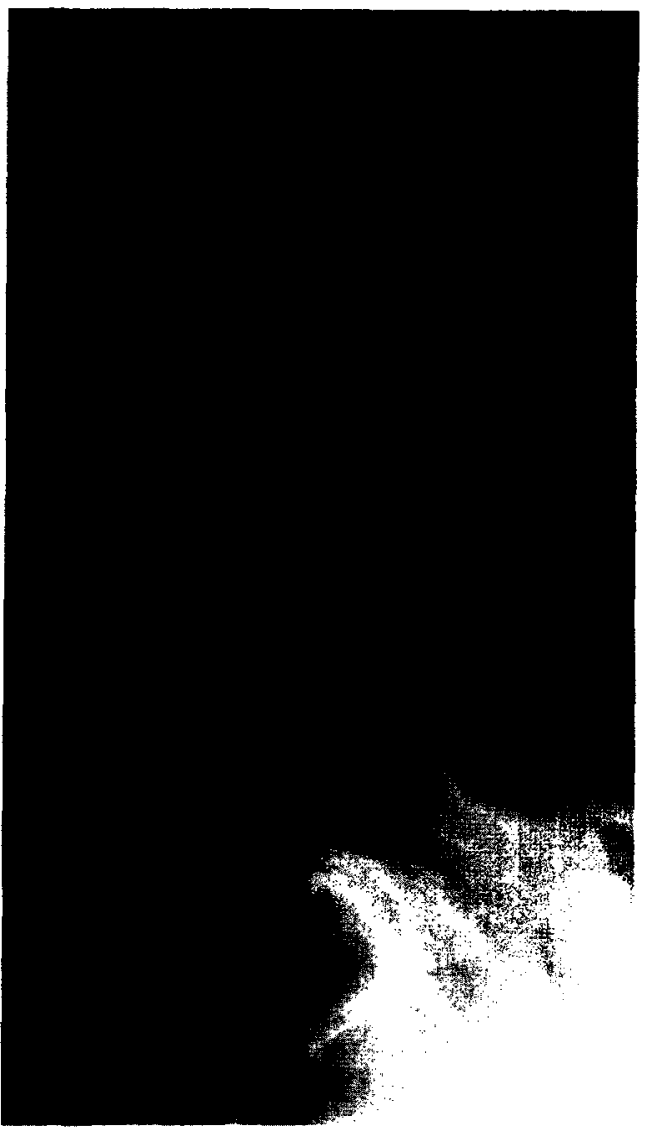

A

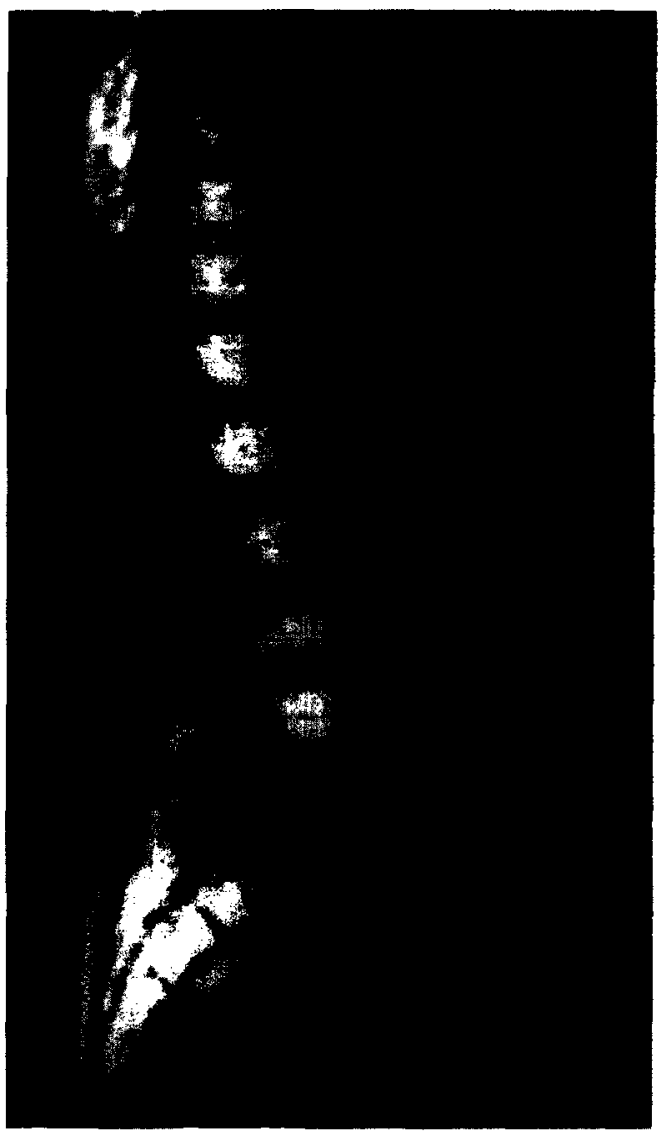

B

Fig. 7. (A) A lateral lumbar spine radiograph of a patient with breast carcinoma shows collapsed upper half of $\mathrm{L} 3$ with diminished L.2-L3 disc space. There is slight loss of height of L5. (B) A sagittal MR section (SE 1030/30) reveals that the marrow fat in $\mathrm{L} 5$ has been replaced by metastatic tumor with marked loss of signal intensity $(\mathrm{SIR}=0.6)$, whereas the collapsed L3 has a normal signal intensity $(\mathrm{SIR}=1.0)$.

study is thus required to examine whether MR has an advantage over the combination of roentgenograms, radionuclide bone scans and $\mathrm{CT}$ in distinguishing malignant from benign collapsed vertebrae.

All the patients with metastatic collapsed vertebrae were examined by the $0.15 \mathrm{~T}$ imager using SE 1030/30. While $T_{1}$ does not totally dominate the signal contrast of the images, the pulse sequence is basically $T_{1}$-weighted. Ten out of eleven with post-traumatic collapsed vertebrae were examined by the $0.35 \mathrm{~T}$ imager using either SE $500 / 28$ or SE $500 / 40$. These two pulse sequences are definitely $T_{1}$-weighted. It is well-documented in the literature that $T_{1}$ contrast decreases with increasing magnetic field strength. ${ }^{4}$ Therefore, the imperfect $T_{1}$-weighted pulse sequence of SE 1030/30 is compensated by the low magnetic field strength used, maintaining the distinction be- tween the two groups of collapsed vertebrae. Had both groups been examined by $0.15 \mathrm{~T}$ imagers using a $T_{1}$-weighted pulse sequence, the distinction between them would have been even greater with SIR being polarized farther away from the dividing value of 0.8 . We do not believe that the good distinction between the malignant and benign collapsed vertebrae was due to the difference in the equipment and technique.

A myelographic effect can be obtained in MR images by an SE pulse sequence using long TR ( 2000 $\mathrm{ms})$ and relatively short TE $(30 \mathrm{~ms})$ intervals. However, our previous experience showed that patients with neurological symptoms and signs, especially pain and parathesia, did not tolerate a long scan time of over 20 minutes. The patients frequently moved to adjust their position in the magnet to ease the symptoms. Although the movement was usually slight dur- 
ing data acquisition, the images were degraded by motion artifacts. Therefore we have not used the myelographic pulse sequence in our study.

\section{REFERENCES}

1. Anderson, R.; Braun, I.F.; Hoffman, J.C. Jr.; Barrow, D.; Davis, P.C.; Jacobs, L.H.; Eisner, R. MR imaging of vertebral body tumors. Radiology 161(P):133; 1986.

2. Daffner, R.H.; Lupetin, A.R.; Dash, N.; Deeb, Z.L.; Sefczek, R.J.; Shapiro, R.L. MRI in the detection of malignant infiltration of bone marrow. A.J.R. 146: 353-358; 1986.

3. Gebarski, S.S.; Gebarski, K.M.S. Myelopathy: clinical and cost effectiveness of MR imaging, myelography, and CT. Radiology 161(P):33; 1986.

4. Johnson, G.A.; Herfkens, J.; Brown, M.A. Tissue relaxation time: in vivo field dependants. Radiology 156: $805-810 ; 1985$.

5. Kagan, R.; eds. Radiologic contribution to cancer management: bone metastases. A.J.R. 147:305-312; 1986.

6. Krol, G.; Heier, L.; Becker, R.; Watson, R.C.; Deck, M.D.F. MR imaging of primary and metastatic tumors of the spine: contribution of $T_{1}$ and $T_{2}$-weighted and multiple echo sequences. Radiology 161(P):220; 1986.

7. Maravilla, K.R.; Weinreb, J.C.; Susis, R.; Nunnally, R.L. Magnetic resonance demonstration of multiple sclerosis plaques in the cervical cord. A.J.R. 144: 381-385; 1985.
8. Masaryk, T.J.; Modic, M.T.; Geisinger, M.A.; Standefer, J.; Hardy, R.W.; Boumphrey, F.; Duchesneau, P.M. Cervical myelopathy: a comparison of magnetic resonance and myelography. J. Comput. Assist. Tomogr. 10(2):184-194; 1986.

9. Modic, M.T.; Masaryk, T.; Boumphrey, F.; Goormastic, M.; Bell, G. Lumbar herniated disk disease and canal stenosis: Prospective evaluation by surface coil MR, CT, and myelography. A.J.R. 147:757-765; 1986.

10. Norman, D.; Mills, C.M.; Brant-7awadski, M.; Yeates, A.; Crooks, L.E.; Kaufman, L. Magnetic resonance imaging of the spinal cord and canal: potentials and limitations. A.J.R. 141:1147-1152; 1983.

11. Quencer, R.M.; Sheldon, J.J.; Post, M.J.D.; Diaz, R.D.; Montalvo, B.M.; Green, B.A.; Eismot, F.J. MRI of the chronically injured cervical spinal cord. A.J.R. 147:125-132; 1986.

12. Slatkin, N.E.; Posner, J.B. Management of spinal epidural metastases. Clin. Neurosurg. 31:698-716; 1983.

13. Sugimura, K.; Yamasaki, K.; Kitagaki, H.; Tanaka, Y.; Kono, M. Bone marrow diseases of the spine: differentiation with $T_{1}$ and $T_{2}$ relaxation times in MR imaging. Radiology 165:541-544; 1987.

14. Tomita, T.; Galicich, J.H.; Sundareson, N. Radiation therapy for spinal epidural metastases with complete block. Acta. Radiol. (Oncol.) 22:135-143; 1983.

15. Utz, J.; Herfkens, R.J.; Fram, E.K.; Woodruff, W.W. III. MR imaging of vertebral metastasis. Radiology 161(P):278; 1986. 